\title{
Atrial standstill
}

INSERM

\section{Source}

INSERM. (1999). Orphanet: an online rare disease and orphan drug data base. Atrial standstill. ORPHA:1344

Atrial standstill is a rare cardiac rhythm disease with a few familial and sporadic cases described to date that is characterized by a transient or permanent absence of electrical and mechanical atrial activity. Electrocardiog raphic findings include bradycardia, ectopic supraventricular rhythms, lack of atrial excitability and absent P waves. 